COMMUNICATIONS IN

ANALYSIS AND GEOMETRY

Volume 4, Number 3, 333-337, 1996

\title{
A Bochner-Hartogs Type Theorem for Coverings of Compact Kähler Manifolds
}

\section{MOHAN RAMACHANDRAN}

Let $M$ be a connected noncompact complex manifold. We say $M$ satisfies Bochner-Hartogs property if $H_{C}^{1}\left(M, \mathcal{O}_{M}\right)=0$. Denote by $C_{C}^{\infty}(M)$ compactly supported smooth functions on $M$ with complex values and by $A_{C}^{0,1}(M)$ compactly supported smooth $(0,1)$ forms on $M$. By Corollary 2.15 of Henkin-Leiterer [5], the condition $H_{C}^{1}\left(M, \mathcal{O}_{M}\right)=0$ is equivalent to the following condition:

Condition BH. For every $\alpha \in A_{C}^{0,1}(M)$ satisfying $\bar{\partial} \alpha=0$, there exists a $u \in$ $C_{C}^{\infty}(M)$ such that $\bar{\partial} u=\alpha$. Henceforth we say that a connected noncompact complex manifold has the Bochner-Hartogs property if the condition $\mathrm{BH}$ is satisfied. See the paper of Harvey-Lawson [4] for more on the Bochner Hartogs property.

Let $\Gamma$ be an infinite group with finite generating set $S$. Without loss of generality we can assume that $S^{-1}=S$.

Define $|x|_{S}=\inf \left\{n: x=g_{1} \cdots g_{n}, g_{i} \in S\right\}$.

Define $\gamma(t)=\#\left(B_{t}\right)$ where $B_{t}=\left\{\left.x \in \Gamma|| x\right|_{S} \leq t\right\}$.

We say $\Gamma$ has more than quadratic growth if there exists constants $C>0$ and $\epsilon>0$ such that

$$
\gamma(t) \geq C t^{2+\epsilon} \text { for } t \geq 1
$$

It follws from a deep theorem of Gromov [8] that $\Gamma$ is either a finite extension of $\mathbb{Z}, \mathbb{Z}^{2}$ or satisfies (1). We now state our main theorem.

Main Theorem. Let $X$ be a connected compact Kähler manifold. Let $\tilde{X}$ be a galois covering of $X$ with infinite covering group $\Gamma$ of more than quadratic growth. If $\tilde{X}$ admits a nonconstant holomorphic function, then either $\tilde{X}$ maps properly onto a Riemann surface or it satisfies the Bochner-Hartogs property.

The restriction on the growth of the group $\Gamma$ is not necessary if $\Gamma$ is the fundamental group of $X$. We say a group $\Gamma^{\prime}$ is commensurable to a group $\Gamma$ if there exists a finite normal subgroup $N$ of $\Gamma^{\prime}$ such that $\Gamma$ is isomorphic to a finite index subgroup of $\Gamma^{\prime} / N$. Combining the above theorem with theorem 3.1 of Gromov [9] we get the following Corollary. 
Corollary 1. Let $X$ be a connected compact Kähler manifold with infinite fundamental group. If the universal cover admits a nonconstant holomorphic function and $\pi_{1}(X)$ is not commensurable to the fundamental group of a compact Riemann surface, then the universal cover satisfies the BochnerHartogs property.

Our assumption of existence of a nonconstant holomorphic function in the corollary is consistent with the Shafarevitch conjecture which states that the universal cover of a smooth projective variety is holomorphically convex. In fact we conjecture that the main theorem is valid without the assumption of existence of nonconstant holomorphic functions.

The proof of Main Theorem consists of two parts. First we solve the equation

$$
\bar{\partial} u=\alpha \quad \text { where } \quad \alpha \in A_{C}^{0,1}(\tilde{X}) \quad \text { and } \quad \bar{\partial} \alpha=0
$$

where $u$ has some decay at infinity on $\tilde{X}$. Then we use a global argument to prove that in case $u$ is not compactly supported $\tilde{X}$ maps properly onto Riemann surface. To solve equation (2) we use results of Varopoulos $[6,7]$ and Chavel-Feldman [3] on estimates for the Green's function of the Laplacian on $\tilde{X}$. It should be remarked here that our proof is in the spirit of Bochner [2].

Acknowledgements. This paper was inspired by a remark of Professor D. Burns that the theorem [1] that universal covers of compact Kähler manifolds have at most one end, should be thought of as an example of Hartogs phenomenon. I would like to thank Professors D. Arapura, P. Bressler, J. Faran and T. Napier for useful conversations.

Let $\Gamma$ be an infinite group with finite generating set $S=S^{-1}$.

Let $\|f\|_{p}=\left[\sum_{g \in \Gamma}|f(g)|^{p}\right]^{\frac{1}{p}}$ where $p \geq 1$ is a real number.

Let $\Delta_{f}(x, y)$ denote

$$
\Delta_{f}(x, y)=|f(x)-f(x y)|^{2} \quad x, y \in \Gamma
$$

and

$$
\|f\|_{D}^{2}=\sum_{x \in \Gamma_{s \in S}} \Delta_{f}(x, s)
$$

where $f$ is complex valued function on $\Gamma$. The space of all complex valued functions on $\Gamma$ with finite support will be denoted by $C_{0}(\Gamma)$. 
Theorem 2. [Varopoulos] If $\Gamma$ has more than quadratic growth, then there exists $a n>2$ and a constant $C>0$ such that

$$
\|f\|_{\frac{2 n}{n-2}} \leq C\|f\|_{D} \quad \text { for every } f \in C_{0}(\Gamma) .
$$

Proof. See [6]. We will assume unless otherwise stated that $\Gamma$ has more than quadratic growth. Combining Theorem 1.1 with Theorem 2 page 475 in Chavel-Feldman [3], we get the following result.

Theorem 3. [Varopoulos, Chavel-Feldman] Let $M$ be a compact connected Riemannian manifold and $\tilde{M}$ a Galois covering of $M$ with Galois group $\Gamma$. Then the Laplacian on $\tilde{M}$ with respect to the pullback metric admits a minimal positive Green's function $G(x, y)$ with the followin property.

There exist constants $C>0, K>0$ and $\nu>2$ such that

$$
|G(x, y)| \leq \frac{C}{d(x, y)^{\nu-2}} \quad \text { for all } \quad x, y \in \tilde{M} \quad \text { with } \quad d(x, y) \geq K .
$$

Here $d$ is the distance function associated to the Riemannian metric. Let $P$ denote the projection of the space of square integrable $(0,1)$ forms into its harmonic part.

Proposition 4. Let $X$ be a connected compact Kähler manifold $\tilde{X}$ a Galois covering with Galois group $\Gamma$. If $\alpha \in A_{C}^{0,1}(\tilde{X})$ such that $\bar{\partial} \alpha=0$ then there is a function $u$ vanishing at infinity such that

$$
\bar{\partial} u=\alpha-P \alpha .
$$

Further $u$ is pluriharmonic outside of a compact set.

Proof. Let $G$ denote the integral operator associated to $2 G(x, y)$. From the Kähler identities it follows that $u=G \bar{\partial}^{*} \alpha$ is a solution of the equation

$$
\bar{\partial} u=\alpha-P \alpha .
$$

where $\bar{\partial}^{*}$ is the formal adjoint of $\bar{\partial}$. By Theorem 2 it follows that $u$ vanishes at infinity since $\bar{\partial}^{*} \alpha$ is compactly supported. Since $\alpha$ is compactly supported and $P \alpha$ is a square integrable harmonic one form it follows that $u$ is pluriharmonic outside the support of $\alpha$. 
Proof. [Main Theorem] Let $h: \tilde{X} \rightarrow \mathbb{C}$ be a nonconstant holomorphic function. Connected components of fibres of a map will be called levels. If a level of $h$ is compact, by remark 3.9 due to Gromov in [1] it follows that all levels of $h$ are compact. By Stein factoring $h$ we get a proper surjective map from $\tilde{X}$ onto a smooth Riemann surface with connected fibres. We now assume that all the levels of $h$ are noncompact. We will show that $\tilde{X}$ satisfies condition BH, We will also assume without loss of generality that $\tilde{X}$ has one end. This is because the number of ends of $\tilde{X}$ is 1,2 , or infinity. $\tilde{X}$ cannot have two ends because this would imply that $\Gamma$ is finite extension of $\mathbb{Z}$. If $\tilde{X}$ had infinitely many ends, then it would map properly onto a Riemann surface by theorem 1.1 and $2.3 \mathrm{c}$ of Gromov [9]. Also see Corollary 4.6 [1]. Let $\alpha \in A_{C}^{0,1}(\tilde{X})$ such that $\bar{\partial} \alpha=0$. By Proposition 1 there is a function $u$ which is pluharmonic outside of a compact set and vanishes at infinity such that

$$
\bar{\partial} u=\alpha-P \alpha .
$$

Since all levels of $h$ are noncompact, $u$ vanishes on the levels of $h$ which disjoint from the support of $\alpha$, by the maximum principle for pluriharmonic functions. Hence, $u$ vanishes on the nonempty set $h^{-1}(\mathbb{C} \backslash h$ (support of $\alpha)$ ).

Since $\tilde{X}$ has one end, $\tilde{X} \backslash$ (support of $\alpha$ has one connected component which is not relatively compact in $\tilde{X}$. Now $u$ vanishes on this component. Therefore the support of $u$ is compact.

Since $u$ and $\alpha$ are compactly supported it follows that $P \alpha$ is a compactly supported harmonic $(0,1)$ form. This implies that $P \alpha$ is identically zero. Therefore $\tilde{X}$ satisfies condition $\mathrm{BH}$. 


\section{References.}

[1] D. Arapura, P. Bressler, and M. Ramachadran(1992) On the fundamental group of a compact Kähler manifold, Duke Math. Journal Vol 68 No.3, 477-488.

[2] S. Bochner (1943)Analytic and Meromorphic continuation by means of Green's formula, Annals of Math, Vol.(2) 44, 652-673.

[3] I. Chavel and E. Feldman(1991)Modified isoperimetric constants, and large time heat diffusion in Riemannian manifolds, Duke Math. Journal,Vol.64 No.3, 473-499.

[4] F.R. Harvey and H.B. Lawson(1975)Boundaries of complex analytic varieties I,Annals of Math, Vol.102, 223-290.

[5] G. Henkin and J. Leiterer(1988)Andreotti-Grauert theory by integral formulas, Birkauser.

[6] N.Th. Varopoulos(1986)Theorie du potential sur des groupes et des varietes, C.R. Acad. Sci. Paris, Vol 302, 203-205.

[7] N.Th. Varopoulos(1987)Random walk and brownian motion on manifold, Symposia Mathematica, Vol.29, Academic Press London, 97-109.

[8] M. Gromov(1981)Groups of polynomial growth and expanding maps, Publicationes Mathematiques IHES, Vol.53, 53-73.

[9] M. Gromov(1989)Sur le groupe fundamental d'une variete Kahlerienne, C.R. Acad. Sci., t308 Ser.I, 67-70.

RECEIVED MARCH 7, 1994.

DePaRTMENT OF MATHEMATICS

SUNY

BUFFALO, NY 14214-3093

E-MAIL: RAMAC-M@NEWTON.MATH.BUFFALO.EDU 